Published in final edited form as:

Plasmid. 2012 July ; 68(1): 33-42. doi:10.1016/j.plasmid.2012.02.003.

\title{
Low copy expression vectors for use in Yersinia sp. and related organisms
}

\author{
Markus W. Obrist ${ }^{1}$ and Virginia L. Miller ${ }^{1,2,{ }^{*}}$ \\ ${ }^{1}$ Department of Genetics, University of North Carolina School of Medicine, Chapel Hill, NC 27599 \\ ${ }^{2}$ Department of Microbiology and Immunology, University of North Carolina School of Medicine, \\ Chapel Hill, NC 27599
}

\section{Abstract}

In Yersinia, the most commonly used expression vectors for genetic studies such as gene complementation do not effectively allow for both induction and repression of gene expression. Additionally, there is no expression system available that can be induced in bacteria growing in vitro as well as in vivo, e.g. in eukaryotic cell lines or in living animal models. Here, we present a series of novel inducible low copy expression vectors that are well suited for use in the Yersinia species. Their tet operator/promoter/repressor system makes them distinct from other vectors, and gene transcription in bacteria can easily be induced by addition of anhydrotetracyline (ATc) either to the growth medium, to tissue culture medium during bacterial infections of cell lines or by injection into animals infected with bacteria. Researchers can choose between two different antibiotic resistances (kanamycin or spectinomycin), between two copy numbers (5 or 12-22) as well as between two different versions for expression from either the native RBS and ATG or RBS and ATG encoded in the plasmid. The whole vector series contains the same multi-cloning site from pBluescript II KS+ that allows for easy subcloning. Moreover, these vectors are built in a modular fashion that makes it simple to adapt them for other purposes. Finally, in addition to their use in Yersinia they are suitable for use in many other Enterobacteriaceae.

\section{Keywords}

complementation vector; tet operator; tetracycline inducible; Yersinia; low copy number

\section{Introduction}

Bacterial genetics often involves complementation studies that rely on inducible promoters to allow for control of expression of the complementing gene. One way to achieve this goal is to design an inducible gene construct that is introduced on the chromosome of a given deletion mutant. As this strategy is often time consuming and sometimes hindered by a limited number of genetic tools for certain bacterial species, plasmid expression vectors have gained huge popularity. However expression vectors have their own drawbacks. They often tend to over-produce the protein of interest because of the high copy number, their

(C) 2012 Elsevier Inc. All rights reserved.

"Corresponding author: Mailing address: Department of Microbiology and Immunology, University of North Carolina at Chapel Hill, 804 Mary Ellen Jones Bldg, Campus Box 7290, Chapel Hill, NC 27599-7290, Phone: 919-966-9956, Fax 919-962-8103, vlmiller@med.unc.edu.

Publisher's Disclaimer: This is a PDF file of an unedited manuscript that has been accepted for publication. As a service to our customers we are providing this early version of the manuscript. The manuscript will undergo copyediting, typesetting, and review of the resulting proof before it is published in its final citable form. Please note that during the production process errors may be discovered which could affect the content, and all legal disclaimers that apply to the journal pertain. 
induction system can be difficult to titrate, some promoters are not fully repressed in the absence of the inducer, and some vectors do not replicate in the species of interest in contrast to Escherichia coli, where most cloning is performed.

Low copy vectors often used for gene complementation in the Yersinia species such as pWSK129, pWKS130, pKW1, pACYC184, pRW50 or pTM100 are based on either the p15A or the pSC101 origin of replication (Chang and Cohen, 1978; Michiels and Cornelis, 1991; Wang and Kushner, 1991; Lodge et al., 1992; Wagner et al., 2008). Several of these vectors contain either the tac or lac promoter which can be repressed by LacI (Jacob and Monod, 1961; de Boer et al., 1983). As none of these vectors contain the lac repressorencoding lacI gene, they rely on the repressor gene to be provided in trans e.g. on a plasmid or on the chromosome. The Yersinia enterocolitica, Yersinia pseudotuberculosis and Yersinia pestis chromosomes do encode the lacI gene but in our experience, their gene products do not efficiently repress the aforementioned promoters. Therefore, these vectors are used under derepressed conditions, and genes cloned into these vectors are constitutively transcribed. An inducible low copy expression vector that has been successfully used in Yersinia is pBAD33 (Guzman et al., 1995; Yamaguchi et al., 2010). The vector contains the ara $B A D$ promoter that can be induced by addition of arabinose to the medium.

Unfortunately, this induction system produces mixed populations under subsaturating arabinose concentrations where some cells are not induced in contrast to others that are fully induced. This result was found due to unequal uptake of arabinose, which does not freely diffuse into the cells but is actively taken up by transport systems which are under arabinose induction themselves (Siegele and Hu, 1997).

Another common expression system uses the tet operator/promoter, which can be induced by the antibiotic tetracyline (Hillen and Berens, 1994). Anhydrotetracyline (ATc) is a tetracyline derivative that also induces the tet operator/promoter system. ATc does not depend on active uptake into either bacterial or eukaryotic cells, binds stronger to the tet repressor and is less toxic to the cells than tetracycline (Gossen and Bujard, 1993; Skerra, 1994; Lutz and Bujard, 1997). Thus, ATc can be used to induce tetracycline inducible promoters in bacteria as well as in eukaryotic cells (Gossen and Bujard, 1993). Recently, the tet operator/promoter system was successfully used to induce expression of a reporter construct in $Y$. pestis cells residing in the lungs of mice by injection of ATc into the animals (Lathem et al., 2007). Therefore, we decided to use the tet operator/promoter system to construct a series of low copy expression vectors. The vector series has a modular basis where the origin of replication and antibiotic resistance can be exchanged for individual purposes. These vectors increase the number of genetic tools for studying gene expression in the Yersinia species as well as in other Enterobacteriaceae.

\section{Materials and methods}

\subsection{Bacterial strains and growth conditions}

Bacterial strains used in this work are presented in Table 1. Escherichia coli strain DH5a was used for cloning purposes and grown at $37^{\circ} \mathrm{C}$ in LB medium (Difco; Sparks, MD). Yersinia enterocolitica strain JB580v was used for control experiments and grown for $15 \mathrm{~h}$ at $26^{\circ} \mathrm{C}$ in LB medium or for $48 \mathrm{~h}$ at $26^{\circ} \mathrm{C}$ on LB plates. Yersinia pseudotuberculosis strain IP32953 and Yersinia pestis strain YP6 were used for control experiments and were grown for $15 \mathrm{~h}$ at $26^{\circ} \mathrm{C}$ in filter sterilized brain heart infusion medium (BHI, Bacto; Sparks, MD) or for $48 \mathrm{~h}$ at $26^{\circ} \mathrm{C}$ on BHI plates (BHI Agar, Bacto; Sparks, MD). Yersinia enterocolitica strain YVM1250 was used to test expression and was grown at $26^{\circ} \mathrm{C}$ in $\mathrm{LB}$ medium. To perform $\beta$-galactosidase tests $Y$. entercolitica strain YVM1250 was grown overnight in Lbroth ( $1 \%$ tryptone, $0.5 \%$ yeast extract; referred to hereafter as LB-0) and subcultured into L-broth containing $290 \mathrm{mM} \mathrm{NaCl}$ (referred to hereafter as LB-290). We noticed that cells 
carrying the new vectors were growing more slowly and forming much smaller colonies when selected with our previously published antibiotics concentrations (Walker and Miller, 2004; 2009; Walker et al., 2010). We therefore lowered concentrations for kanamycin and spectinomycin. For plasmid selection in E. coli, antibiotics (all from Sigma-Aldrich Corp.; St. Louis, MO) were added at the following concentrations: ampicillin, $100 \mu \mathrm{g} / \mathrm{ml}$; chloramphenicol, $25 \mu \mathrm{g} / \mathrm{ml}$; kanamycin, $25 \mu \mathrm{g} / \mathrm{ml}$; spectinomycin, $50 \mu \mathrm{g} / \mathrm{ml}$. For selection of plasmids in the Yersinia species antibiotics were added at the following concentrations: chloramphenicol, $12.5 \mu \mathrm{g} / \mathrm{ml}$; kanamycin, $50 \mu \mathrm{g} / \mathrm{ml}$; spectinomycin, $50 \mu \mathrm{g} / \mathrm{ml}$.

\subsection{DNA isolation, manipulation and oligonucleotides}

Genomic DNA was isolated from $Y$. enterocolitica strain JB580v according to standard protocols (Sambrook and Russell, 2001). Plasmid DNA was isolated from E. coli strains using Wizard Plus SV Miniprep (Promega; Madison, WI). DNA extraction from agarose gels was performed using Wizard SV Gel and PCR Clean-Up System (Promega). DNA manipulations were carried out according to standard procedures (Sambrook and Russell, 2001) unless otherwise stated. Restriction endonucleases and T4 DNA ligase were purchased from New England Biolabs (NEB; Ipswich, MA) and were used as recommended by the supplier. Cloned Pfu DNA polymerase was purchased from Agilent Technologies Inc. (Santa Clara, CA). Chemically competent $E$. coli cells were made according to the rubidium chloride method (NEB protocol) and were transformed by heat-shock. Competent $Y$. enterocolitica, $Y$. pseudotuberculosis and $Y$. pestis cells were prepared as described in (Walker and Miller 2004) and transformed by electroporation (BIORAD Gene Pulser II; settings: $1.8 \mathrm{kV}, 25 \Omega, 400 \mathrm{~mA}$ ). Oligonucleotides used in this study are listed in Table 2 and were purchased from Integrated DNA Technologies (IDT; Coralville, IA). DNA sequence analysis was performed by either Eton Bioscience Inc. (San Diego, CA) or GENEWIZ (South Plainfield, NJ).

\subsection{Plasmid construction}

Vectors constructed in this study are presented in Results and are listed in Table 3. Plasmids used and constructed in this study are listed in Table 1. All plasmids were confirmed by restriction digestion as well as by sequencing of the critical regions. To test the newly constructed vector pMWO-005, a derivative plasmid, pMWO-038, encoding 6xHis-ysr $R$ was constructed. The $y s r R$ gene was PCR amplified from $Y$. enterocolitica genomic DNA using MWO-147 and MWO-072. MWO-147 adds a 6xHis-tag encoding sequence to the 5' end of the $y s r R$ gene. The PCR product was digested with $K p n I$ and $X b a I$ and ligated into pMWO-005. As we had set the start codon too far from the RBS, we saw almost no expression from pMWO-038 (data not shown). Therefore, we designed a new forward primer, MWO-150, using the same restriction site. Using pMWO-038 as template, 6xHis$y s r R$ was PCR amplified with MWO-150 and MWO-072. The PCR product was digested with $K p n I$ and $X b a I$ and ligated into pMWO-005 thereby creating pMWO-081. The insert was sequenced with MWO-fw and MWO-rv-1.

\subsection{ATc toxicity test}

Y. enterocolitica (JB580v), Y. pseudotuberculosis (IP32953) and Y. pestis (YP6) were grown overnight at $26^{\circ} \mathrm{C}$. The next morning, two subcultures for each strain were inoculated to an optical density at $600 \mathrm{~nm}\left(\mathrm{OD}_{600}\right) \approx 0.2$. To one culture of each pair of strains, anhydrotetracycline hydrochloride [ATc (Fluka Analytical; Sigma-Aldrich)] was added using the following concentrations: 1.0 or $2.0 \mu \mathrm{g} / \mathrm{ml}$ and additionally in the case of $Y$. enterocolitica: $0.1,0.25,0.5,0.75 \mu \mathrm{g} / \mathrm{ml}$. Cultures were grown at $26^{\circ} \mathrm{C}$ and optical densities were checked after $8 \mathrm{~h}$. 


\subsection{Growth curve experiment}

$Y$. enterocolitica, $Y$. pseudotuberculosis and $Y$. pestis harboring either pMWO-005, pMWO-073, pMWO-074 or pMWO-075 were grown overnight at $26^{\circ} \mathrm{C}$ with antibiotic selection. The next morning, one subculture for each strain was subcultured to $\mathrm{OD}_{600} \approx 0.2$ and grown at $26^{\circ} \mathrm{C}$ under antibiotic selection up to $\mathrm{OD}_{600} \approx 0.4$. Then each culture was split into two subcultures and one was induced with $50 \mathrm{ng} / \mathrm{ml} \mathrm{ATc}$. Cells were grown for $6 \mathrm{~h}$ and optical densities were monitored each hour.

\subsection{Plasmid stability test}

$Y$. enterocolitica, $Y$. pseudotuberculosis and $Y$. pestis harboring either pMWO-005, pMWO-073, pMWO-074 or pMWO-075 were grown overnight at $26^{\circ} \mathrm{C}$ with antibiotic selection. The next morning, two subcultures for each strain were subcultured to $\mathrm{OD}_{600} \approx$ 0.2 . One culture of each strain pair was grown with and one without antibiotic selection. Cells were grown for $6 \mathrm{~h}$. Samples were taken and serial dilutions were made and plated onto LB or BHI plates with or without antibiotics. Plates were incubated for $48 \mathrm{~h}$ at $26^{\circ} \mathrm{C}$, colonies were counted and numbers were analyzed and compared.

\subsection{Protein expression test and Western blot}

pMWO-081 was transformed into YVM1250 by electroporation and transformants were grown on selective $\mathrm{LB}$ agar plates at $26^{\circ} \mathrm{C}$ for two days. Five ml LB cultures containing 20 $\mu \mathrm{g} / \mathrm{ml}$ nalidixic acid and $100 \mu \mathrm{g} / \mathrm{ml}$ kanamycin were inoculated with a single colony and grown overnight at $26^{\circ} \mathrm{C}$ on a roller drum. These cultures were subcultured to $\mathrm{OD}_{600} \approx 0.2$ into $2 \mathrm{ml}$ fresh medium with antibiotics. Cultures were grown at $26^{\circ} \mathrm{C}$ on a roller drum until $\mathrm{OD}_{600} \approx 0.4$, where they were induced with $50 \mathrm{ng} / \mathrm{ml} \mathrm{ATc}$ and grown for $2 \mathrm{~h}$ under the same conditions. Initially, we also tested other concentrations but found $50 \mathrm{ng} / \mathrm{ml}$ ATc to be the optimal induction level. $\mathrm{OD}_{600}$ was read and $100 \mu 1$ samples of cultures were harvested and pelleted. Pelleted cells were resuspended in 5x SDS-PAGE sample buffer to $1 \mathrm{ml}$ per $\mathrm{OD}_{600}$ $=1.0$. Samples of 0.10 OD were analyzed by SDS-PAGE on 16\% tricine gels (Schägger and Jagow, 1987) and transferred onto nitrocellulose membranes by Western blot. His-YsrR was detected using an anti-His antibody (monoclonal mouse anti-His antibody; $0.1 \mu \mathrm{g} / \mathrm{ml}$; THE $^{\text {TM }}$ His Tag Antibody; GenScript; Piscataway, NJ) and a secondary antibody [goat-antimouse IgG (Fc specific)-peroxidase; 1:50,000 dilution; Sigma-Aldrich], followed by chemiluminescence detection (Amersham ${ }^{\mathrm{TM}}$ ECL $^{\mathrm{TM}}$ Western Blotting Detection Reagents; GE Healthcare; Waukesha, WI).

\section{$2.8 \beta$-galactosidase assay}

Prior to each $\beta$-galactosidase test, YVM1250 was transformed with the appropriate plasmids by electroporation and colonies were grown on LB-agar plates for two days at $26^{\circ} \mathrm{C}$. Saturated cultures grown overnight at $26^{\circ} \mathrm{C}$ on a roller drum in LB-0 were diluted into fresh LB-290 to $\mathrm{OD}_{600}$ of 0.2 and grown for $2 \mathrm{~h}$ with or without $50 \mathrm{ng} / \mathrm{ml}$ ATc using the same growth conditions. Antibiotics were added as necessary to retain plasmids. After $2 \mathrm{~h}$, cultures were put immediately on ice to prevent further growth and $\beta$-galactosidase assay was performed as described before (Miller, 1992).

\section{Results and discussion}

\subsection{Design and characteristics of new vectors}

The vector series presented in this study is intended for gene complementation, low level protein expression and related purposes. We designed the vectors to have the following characteristics: (i) replicate in the Yersinia species ( $Y$. enterocolitica, Y. pseudotuberculosis and $Y$. pestis) as well as in E. coli, (ii) have a low copy origin of replication, (iii) bear a 
selection marker suitable in all mentioned organisms, (iv) contain a versatile multi-cloning site, (v) have a tightly controlled inducible promoter, (vi) have vector derivatives suited for genes that require expression from the native RBS and translational start codon and (vii) are built in a modular fashion, to allow for future adaptations.

To fit these criteria we chose the pSC101 as well as the p15A origin of replication. Both origins of replication have been shown to replicate in E. coli as well as in $Y$. enterocolitica, Y. pseudotuberculosis and Y. pestis (Cozzarelli et al., 1968; Cohen et al., 1973; Pierson and Falkow, 1993; Pepe et al., 1995; Jackson et al., 2004; Heroven and Dersch, 2006; Lenz et al., 2011). For complementation studies to succeed, it is desirable not to overexpress the gene of interest. Balancing between lowest possible copy number and cloning practicalities, the pSC101 and the p15A origin of replication seemed to be an ideal fit. Plasmids bearing the pSC101 origin of replication were shown to have about 5 copies per cell (Cabello et al., 1976; Stoker et al., 1982) whereas plasmids carrying the p15A origin of replication were shown to generate between 12-22 copies per cell (Cozzarelli et al., 1968; Chang and Cohen, 1978).

With regard to natural antimicrobial susceptibility (Martins et al., 1998; Galimand et al., 2006) Y. pseudotuberculosis and $Y$. pestis are susceptible to most antimicrobials (Martins et al., 1998; Galimand et al., 2006), but $Y$. enterocolitica strains are often naturally resistant to some B-lactams, whose derivatives, e.g. ampicillin or carbenicillin, are commonly used in laboratories (Stock and Wiedemann, 1999). Therefore, we chose to use kanamycin and spectinomycin resistance genes for our vectors. To be able to choose a variety of cloning strategies, we selected the multi-cloning site present in pWKS130, which originates from pBluescript II KS+ (Alting-Mees and Short, 1989; Wang and Kushner, 1991). As this multicloning site is present in all vectors, genes of interest can be easily subcloned from one vector to another.

Controlled induction of gene transcription is important and we therefore chose to use the tetracyline inducible promoter/operator/repressor system reviewed in (Hillen and Berens, 1994). In particular we chose to use a promoter derivative that combines the promoter of phage lambda with the operator of the tet operon (Tn 10) (Lutz and Bujard, 1997). A tetracycline derivative, ATc, has been shown to diffuse freely into bacterial and eukaryotic cells without being dependent on an active uptake system (Gossen and Bujard, 1993). This allows induction of gene transcription in bacteria which reside in either eukaryotic cells or within living animals (Lathem et al., 2007). Furthermore, ATc is less toxic and binds tighter to the tet repressor than tetracycline (Gossen and Bujard, 1993).

As membrane protein encoding genes and some other genes often include special leader sequences, we designed some vector derivative, which contain neither the RBS nor the ATG start codon at the beginning of the multi-cloning site. In addition, we designed this vector series in a modular fashion where the antibiotic resistance as well as the origin of replication can be easily exchanged for future adaptations.

\subsection{Sequencing of pWKS130}

As we intended to use pWKS130 as a source for the pSC101 origin of replication as well as for the multi-cloning site, we sequenced this vector by primer walking using primers MWO-010 to MWO-036 (Table 2). When assembling the sequences, we noticed that the flori-lacZa fragment has the opposite orientation as published (Wang and Kushner, 1991). This finding was confirmed by restriction analysis (data not shown). The sequence of pWKS130, starting with the BgII site, was deposited in GenBank (accession number JQ283951). 


\subsection{Construction of pMWO-005 and pMWO-034}

In a first step, the pSC101 origin of replication and the kanamycin resistance gene were combined. For this purpose, the $k a n R$ gene was PCR amplified from the pET24b+ vector using primers MWO-042 and MWO-043 (PCR \#1, Fig. 1A). Primer MWO-042 anneals upstream of the kanR promoter and adds two unique restriction sites, MluI and NheI. MWO-043 anneals at the $3^{\prime}$-end of $k a n R$ and introduces a 26 bp overlapping sequence at the $3^{\prime}$-end of the PCR \#1 product. To prevent read-through from the $k a n R$ gene the $r r n B$ T1 transcription terminator (Brosius et al., 1981; Brosius, 1984) was amplified from E. coli BL21PRO genomic DNA using primers MWO-044 and MWO-045 (PCR \#2, Fig. 1A). MWO-044 adds the corresponding 26 bp overlapping sequence of MWO-043 at the $5^{\prime}$-end of the $r r n B$ T1 terminator and primer MWO-045 adds two unique restriction sites, $S p h$ and PvuII, to the $3^{\prime}$-end of the product of PCR \#2. The products of PCR \#1 and \#2 were then fused using the overlap PCR technique (Horton et al., 1989) with the products as templates and MWO-042 and MWO-045 as primers (PCR \#3, Fig. 1A). Vector pWKS130 was digested with Nael resulting in five fragments of different length, each having blunt ends. The 2008bp DNA fragment containing the pSC101 origin of replication was isolated and blunt-end ligated with PCR product \#3 (Fig. 2A) creating pStep1 (Fig. 2B). The orientation of ligated fragments was determined by restriction analysis.

In a second step, the tetR gene was introduced into pStep1. For this purpose, three elements were combined: (i) the promoter $\mathrm{P}_{\mathrm{N} 25}$ from coliphage $\mathrm{T} 5$, which is a very strong and constitutive transcribed promoter (Deuschle et al., 1986), (ii) the tet repressor gene, tet $R$, (Hillen and Berens, 1994) and (iii) the $r r n B$ T1 transcription terminator (Brosius et al., 1981; Brosius, 1984). On the chromosome of E. coli strain DH5aPRO, promoter $\mathrm{P}_{\mathrm{N} 25}$ and tetR are already combined. To avoid carrying over the restriction sites, EcoRI and $X b a I$, which are present at the $5^{\prime}$-end of tetR, primers MWO-047 and MWO-048 carry two point mutations that abolish these restriction sites. Additionally, these two primers overlap by $33 \mathrm{bp}$. $\mathrm{P}_{\mathrm{N} 25}$ was amplified using primers MWO-046 and MWO-047, and tet $R$ was amplified with primers MWO-048 and MWO-049 (PCR \#5), both from E. coli DH5aPRO genomic DNA. To prevent read-through from the tetR gene the $\operatorname{rnn} B \mathrm{~T} 1$ transcription terminator was amplified from E. coli BL21PRO genomic DNA using primers MWO-050 and MWO-051 (PCR \#6). Primers MWO-049 and MWO-050 overlap by 32 bp. The products of PCR \#4, \#5 and \#6 where fused using overlap PCR (PCR \#7, Fig. 1B) with primers MWO-046 and MWO-051, which add $S p h \mathrm{I}$ and $P v u I I$ restriction sites to the ends of the product. pStep1 and the product from PCR \#7 were digested with $S p h \mathrm{I}$ and $P v u I I$ and ligated, generating pStep2 (Fig. 2B).

The third step completed the first vector construction by adding the sequence for promoter, operator and multi-cloning site to pStep2. A single fragment containing the strong phage lambda promoter (Deuschle et al., 1986) and the operator $t e t \mathrm{O}_{2}$ from the tet operon (reviewed in (Hillen and Berens, 1994)) present in expression vector pLP-PROTet-6xHN (Lutz and Bujard, 1997) was PCR amplified using primers MWO-052 and MWO-053 (PCR \#8, Fig. 1C). Also, the versatile multicloning site from pBluescript II KS+ was amplified from vector pWKS130 using primers MWO-054 and MWO-055 (PCR \#9, Fig. 1C). Primers MWO-053 and MWO-054 overlap by 22 bp. The products of PCR \#8 and \#9 were fused by overlap PCR using primers MWO-052 and MWO-055 that add NheI and MluI restriction sites to the product (PCR \#10, Fig. 1C). pStep2 and the product from PCR \#10 were digested using NheI and MluI and ligated, generating pMWO-005 (Fig. 2C). The promoter, operator and multi-cloning site encoding sequence are shown in Fig. 3A. Vector pMWO-005 has already been successfully used in our lab to express autotransporterencoding genes from $Y$. pestis (Lenz et al., 2011). 
To construct a derivative of pMWO-005 that is ideal for cloning membrane protein encoding genes, the RBS and the ATG encoding sequences were deleted. For this purpose, the promoter and operator sequences were PCR amplified from vector pMWO-005 with primers MWO-085 and MWO-086 (PCR \#11, Fig. 1C). The multi-cloning site was amplified using primers MWO-087 and MWO-088 with pMWO-005 as template (PCR \#12, Fig. 1C). Primers MWO-086 and MWO-088 each encode a KpnI site. To prepare for combination of the required fragments, PCR product \#11 was digested with NheI and KpnI, PCR product \#12 was digested with $K p n I$ and MluI and vector pMWO-005 was digested with NheI and MluI. Purified fragments were ligated in a three-piece ligation, generating pMWO-034 (Fig. $2 \mathrm{C})$. This shortened version of promoter, operator and multi-cloning site sequence is shown in Fig. 3B.

\subsection{Construction of pMWO-073 - pMWO-078}

To have more options we expanded the vector series by constructing several derivatives. Making use of the modular design of vectors pMWO-005 and pMWO-034 (Fig. 2) we either alone, or together, exchanged the antibiotic resistance and the origin of replication. The gene encoding spectinomycin resistance was PCR amplified using primers MWO-139 and MWO-140 from vector p34S-Sm2 (Dennis and Zylstra, 1998). The primers add NheI and $S p h$ sites to the PCR product. Both vectors and the spectinomycin gene PCR product were digested with NheI and SphI. Purified vector backbones from pMWO-005 and pMWO-034 were each ligated with the spectinomycin resistance insert, giving rise to pMWO-073 and pMWO-076. By changing the origin of replication from pSC101 to p15A it is possible to choose a different copy number, 5 vs. 12-22 respectively (Cozzarelli et al., 1968; Cabello et al., 1976; Chang and Cohen, 1978; Stoker et al., 1982). The origin of replication p15A was PCR amplified from vector pACYC184 (Chang and Cohen, 1978) using primers MWO-137 and MWO-138. MluI and PvuII sites were added to the product by the primers. The vectors and the p15A PCR product were digested with MluI and PvuII. Purified vector fragments without origin were ligated with the new P15A origin of replication insert, generating pMWO-074 and pMWO-077. To construct versions where both antibiotic resistance and origin of replication are exchanged, spectinomycin resistance was introduced into pMWO-074 and pMWO-077 as described above using NheI and SphI. The resulting vectors were named pMWO-075 and pMWO-078 respectively. All vectors constructed in this study are summarized in Table 3 with their respective characteristics. Sequences of new expression vectors, starting with the NheI site, were deposited in GenBank and accession numbers are listed in Table 3.

\subsection{Control tests with vectors}

To make sure that the newly generated vectors do not negatively affect the bacterial cells they are used with, we performed control tests regarding toxicity of the inducer ATc, growth behaviour of cells and plasmid stability. Toxicity of the inducer ATc was tested by growing wild type strains of $Y$. enterocolitica (JB580v), Y. pseudotuberculosis (IP32953) and $Y$. pestis (YP6) with and without the presence of different concentrations of ATc as described in Materials and Methods. $Y$. enterocolitica was found to be resistant up to $0.5 \mu \mathrm{g} / \mathrm{ml}, Y$. pseudotuberculosis up to $1.0 \mu \mathrm{g} / \mathrm{ml}$ and $Y$. pestis up to $2.0 \mu \mathrm{g} / \mathrm{ml} \mathrm{ATc}$, which confirmed previous findings (Lathem et al., 2007). These threshold levels of ATc are well above the working concentration of $0.05 \mu \mathrm{g} / \mathrm{ml}$ that we typically use for induction.

Growth behaviour with or without addition of ATc of Yersinia wild type strains mentioned above harboring either pMWO-005, pMWO-073, pMWO-074 or pMWO-075 was assessed as described in Materials and Methods. This vector subset includes one vector of each combinations of origin of replication and antibiotic resistance. None of the tested strain pairs showed any growth defect (results not shown). 
Plasmid stability was tested as described in Materials and Methods using the same strain and vector selection mentioned above. No plasmid loss was detected with or without antibiotic selection over the tested time ( $6 \mathrm{~h}$ ) (results not shown). Taken together, at the recommended $\mathrm{ATc}$ and antibiotic concentrations in vitro at $26^{\circ} \mathrm{C}$, we could not detect any toxicity towards the cells, impact on growth nor plasmid instability.

\subsection{Test expression of His-YsrR in Yersinia enterocolitica}

To test the efficacy of the promoter-operator system present in this vector series, we cloned the gene sequence encoding the $\mathrm{N}$-terminally $6 x$ His-tagged response regulator, $\mathrm{YsrR}$, into pMWO-005 as described in Materials and Methods. The new plasmid, pMWO-081, was transformed into $Y$. enterocolitica strain YVM1250, which is a ysrR null mutant (Walker et al., 2010). Induction of expression of His-YsrR, a $27 \mathrm{kDa}$ protein, was tested with and without $50 \mathrm{ng} / \mathrm{ml}$ inducer (ATc) and samples were analyzed using SDS-PAGE and detection with anti-His antibodies as described in Materials and Methods. His-YsrR was detected under inducing conditions but absent under repressing conditions (Fig. 4A). If any His-YsrR was expressed, it was below the detection limit of the Western blot. As a further test, we performed $\beta$-galactosidase assays for YsrR function with YVM1250. This strain has a chromosomally integrated $y s a E-l a c Z$ reporter fusion, which is activated by YsrR (Walker et al., 2010) as well as His-YsrR. Expression from the $y s a E$ promoter is activated $\sim 180$-fold after ATc induction compared to the $\Delta y s r R$ mutant carrying the vector pMWO-005 (Fig. 4B). Without ATc induction, the $\triangle y s r R$ mutant carrying pMWO-081 typically showed 6 to 9-fold higher levels of $y s a E-l a c Z$ expression compared to the $\Delta y s r R$ mutant alone or the $\triangle y s r R$ mutant carrying the vector. As the readout of the $\beta$-galactosidase assay is amplified by the fact that YsrR is a transcriptional activator (Walker and Miller, 2004) and the stability of the $\beta$-galactosidase enzyme (Bachmair et al., 1986), it is likely that promoter repression by TetR is nearly complete and only a few molecules of His-YsrR are present during uninduced conditions. Therefore, this inducible vector series should be suitable for most experiments. If complete repression is required, such as for cloning of genes encoding toxic proteins, an additional tet $R$ copy can be added in trans either on a plasmid or on the chromosome.

\section{Conclusion}

In this study, we constructed a series of versatile low copy expression vectors well-suited for use in the Yersinia species. The tet operator/promoter system makes them distinct from other vectors frequently used in Yersinia, and it allows for control of gene expression not only in vitro and potentially also in vivo. As the same multi-cloning site is present in all vectors, genes of interest can be easily subcloned from one vector to another. Vectors can be chosen according to antibiotic selection, copy number and or special needs for use of the native RBS and translational start site. Further, the modular design of these vectors makes them easily adapted for other purposes. And last but not least, it should be possible to use these vectors for gene expression in many different species within the Enterobacteriaceae.

\section{Acknowledgments}

We thank Kimberly A. Walker for critical reading of the manuscript and for stimulating discussions. We thank Ralph Isberg for the generous gift of Yersinia pseudotuberculosis strain IP32953. This research was supported by National Institutes of Health grant AI063299 awarded to V. L. Miller.

\section{References}

Alting-Mees MA, Short JM. pBluescript II: gene mapping vectors. Nucleic Acids Res. 1989; 17:9494. [PubMed: 2555794] 
Bachmair A, Finley D, Varshavsky A. In vivo half-life of a protein is a function of its amino-terminal residue. Science. 1986; 234:179-186. [PubMed: 3018930]

Brosius J. Toxicity of an overproduced foreign gene product in Escherichia coli and its use in plasmid vectors for the selection of transcription terminators. Gene. 1984; 27:161-172. [PubMed: 6202587]

Brosius J, Dull TJ, Sleeter DD, Noller HF. Gene organization and primary structure of a ribosomal RNA operon from Escherichia coli. J Mol Biol. 1981; 148:107-127. [PubMed: 7028991]

Cabello F, Timmis K, Cohen SN. Replication control in a composite plasmid constructed by in vitro linkage of two distinct replicons. Nature. 1976; 259:285-290. [PubMed: 765836]

Cathelyn JS, Crosby SD, Lathem WW, Goldman WE, Miller VL. RovA, a global regulator of Yersinia pestis, specifically required for bubonic plague. Proc Natl Acad Sci USA. 2006; 103:13514-13519. [PubMed: 16938880]

Chain PS, Carniel E, Larimer FW, Lamerdin J, Stoutland PO, Regala WM, Georgescu AM, Vergez LM, Land ML, Motin VL, Brubaker RR, Fowler J, Hinnebusch J, Marceau M, Medigue C, Simonet M, Chenal-Francisque V, Souza B, Dacheux D, Elliott JM, Derbise A, Hauser LJ, Garcia E. Insights into the evolution of Yersinia pestis through whole-genome comparison with Yersinia pseudotuberculosis. Proc Natl Acad Sci USA. 2004; 101:13826-13831. [PubMed: 15358858]

Chang AC, Cohen SN. Construction and characterization of amplifiable multicopy DNA cloning vehicles derived from the P15A cryptic miniplasmid. J Bacteriol. 1978; 134:1141-1156. [PubMed: 149110]

Cohen SN, Chang AC, Boyer HW, Helling RB. Construction of biologically functional bacterial plasmids in vitro. Proc Natl Acad Sci USA. 1973; 70:3240-3244. [PubMed: 4594039]

Cozzarelli NR, Kelly RB, Kornberg A. A minute circular DNA from Escherichia coli 15. Proc Natl Acad Sci USA. 1968; 60:992-999. [PubMed: 4875809]

de Boer HA, Comstock LJ, Vasser M. The tac promoter: a functional hybrid derived from the trp and lac promoters. Proc Natl Acad Sci USA. 1983; 80:21-25. [PubMed: 6337371]

Dennis JJ, Zylstra GJ. Plasposons: modular self-cloning minitransposon derivatives for rapid genetic analysis of gram-negative bacterial genomes. Appl Environ Microbiol. 1998; 64:2710-2715. [PubMed: 9647854]

Deuschle U, Kammerer W, Gentz R, Bujard H. Promoters of Escherichia coli: a hierarchy of in vivo strength indicates alternate structures. EMBO J. 1986; 5:2987-2994. [PubMed: 3539589]

Galimand M, Carniel E, Courvalin P. Resistance of Yersinia pestis to antimicrobial agents. Antimicrob. Agents Chemother. 2006; 50:3233-3236. [PubMed: 17005799]

Gossen M, Bujard H. Anhydrotetracycline, a novel effector for tetracycline controlled gene expression systems in eukaryotic cells. Nucleic Acids Res. 1993; 21:4411-4412. [PubMed: 8415012]

Guzman LM, Belin D, Carson MJ, Beckwith J. Tight regulation, modulation, and high-level expression by vectors containing the arabinose PBAD promoter. J Bacteriol. 1995; 177:41214130. [PubMed: 7608087]

Heroven AK, Dersch P. RovM, a novel LysR-type regulator of the virulence activator gene rovA, controls cell invasion, virulence and motility of Yersinia pseudotuberculosis. Mol Microbiol. 2006; 62:1469-1483. [PubMed: 17074075]

Hillen W, Berens C. Mechanisms underlying expression of Tn10 encoded tetracycline resistance. Annu Rev Microbiol. 1994; 48:345-369. [PubMed: 7826010]

Horton RM, Hunt HD, Ho SN, Pullen JK, Pease LR. Engineering hybrid genes without the use of restriction enzymes: gene splicing by overlap extension. Gene. 1989; 77:61-68. [PubMed: 2744488]

Jackson MW, Silva-Herzog E, Plano GV. The ATP-dependent ClpXP and Lon proteases regulate expression of the Yersinia pestis type III secretion system via regulated proteolysis of YmoA, a small histone-like protein. Mol Microbiol. 2004; 54:1364-1378. [PubMed: 15554975]

Jacob F, Monod J. Genetic regulatory mechanisms in the synthesis of proteins. J Mol Biol. 1961; 3:318-356. [PubMed: 13718526]

Lathem WW, Price PA, Miller VL, Goldman WE. A plasminogen-activating protease specifically controls the development of primary pneumonic plague. Science. 2007; 315:509-513. [PubMed: 17255510] 
Lenz JD, Lawrenz MB, Cotter DG, Lane MC, Gonzalez RJ, Palacios M, Miller VL. Expression during host infection and localization of Yersinia pestis autotransporter proteins (Yaps). J Bacteriol. 2011:5936-5949. [PubMed: 21873491]

Lodge J, Fear J, Busby S, Gunasekaran P, Kamini NR. Broad host range plasmids carrying the Escherichia coli lactose and galactose operons. FEMS Microbiol Lett. 1992; 95:271-276. [PubMed: 1526459]

Lutz R, Bujard H. Independent and tight regulation of transcriptional units in Escherichia coli via the LacR/O, the TetR/O and AraC/I1-I2 regulatory elements. Nucleic Acids Res. 1997; 25:12031210. [PubMed: 9092630]

Martins CH, Bauab TM, Falcão DP. Characteristics of Yersinia pseudotuberculosis isolated from animals in Brazil. J. Appl. Microbiol. 1998; 85:703-707. [PubMed: 9812382]

Michiels T, Cornelis GR. Secretion of hybrid proteins by the Yersinia Yop export system. J Bacteriol. 1991; 173:1677-1685. [PubMed: 1999387]

Miller, JH. A short course in bacterial genetics, A Laboratory Manual and Handbook for Escherichia coli and Related Bacteria. Cold Spring Harbor Laboratory Press; Cold Spring Harbor, NY: 1992.

Pepe JC, Wachtel MR, Wagar E, Miller VL. Pathogenesis of defined invasion mutants of Yersinia enterocolitica in a BALB/c mouse model of infection. Infect Immun. 1995; 63:4837-4848. [PubMed: 7591144]

Pierson DE, Falkow S. The ail gene of Yersinia enterocolitica has a role in the ability of the organism to survive serum killing. Infect Immun. 1993; 61:1846-1852. [PubMed: 7682996]

Sambrook, J.; Russell, DW. Molecular cloning: a laboratory manual. 3rd ed. Cold Spring Harbor Laboratory Press; Cold Spring Harbor, NY: 2001.

Schägger H, Jagow, Von G. Tricine-sodium dodecyl sulfate-polyacrylamide gel electrophoresis for the separation of proteins in the range from 1 to $100 \mathrm{kDa}$. Anal Biochem. 1987; 166:368-379. [PubMed: 2449095]

Siegele DA, Hu JC. Gene expression from plasmids containing the araBAD promoter at subsaturating inducer concentrations represents mixed populations. Proc Natl Acad Sci USA. 1997; 94:81688172. [PubMed: 9223333]

Skerra A. Use of the tetracycline promoter for the tightly regulated production of a murine antibody fragment in Escherichia coli. Gene. 1994; 151:131-135. [PubMed: 7828861]

Stock I, Wiedemann B. An in-vitro study of the antimicrobial susceptibilities of Yersinia enterocolitica and the definition of a database. J. Antimicrob. Chemother. 1999; 43:37-45. [PubMed: 10381099]

Stoker NG, Fairweather NF, Spratt BG. Versatile low-copy-number plasmid vectors for cloning in Escherichia coli. Gene. 1982; 18:335-341. [PubMed: 6290337]

Wagner K, Schilling J, Fälker S, Schmidt M, Heusipp G. A regulatory network controls the expression of the in vivo expressed HreP protease of Yersinia enterocolitica. J Bacteriol. 2008:1666-1676. [PubMed: 19114497]

Walker K, Miller VL. Synchronous Gene Expression of the Yersinia enterocolitica Ysa Type III Secretion System and its Effectors. J Bacteriol. 2009

Walker KA, Miller VL. Regulation of the Ysa type III secretion system of Yersinia enterocolitica by YsaE/SycB and YsrS/YsrR. J Bacteriol. 2004; 186:4056-4066. [PubMed: 15205407]

Walker KA, Obrist MW, Mildiner-Earley S, Miller VL. Identification of YsrT and evidence that YsrRST constitute a unique phosphorelay system in Yersinia enterocolitica. J Bacteriol. 2010; 192:5887-5897. [PubMed: 20870771]

Wang RF, Kushner SR. Construction of versatile low-copy-number vectors for cloning, sequencing and gene expression in Escherichia coli. Gene. 1991; 100:195-199. [PubMed: 2055470]

Yamaguchi S, Gueguen E, Horstman NK, Darwin AJ. Membrane association of PspA depends on activation of the phage-shock-protein response in Yersinia enterocolitica. Mol Microbiol. 2010; 78:429-443. [PubMed: 20979344] 


\section{Highlights}

Construction of small vectors for use in Yersinia sp. and other Enterobacteriaceae Vectors are low-copy (pSC101 ori or p15A ori) and share pBluescript II KS+ MCS

Use of the tet operator allows highly regulated control of expression

Vectors contain either kanamycin or spectinomycin resistance 
A

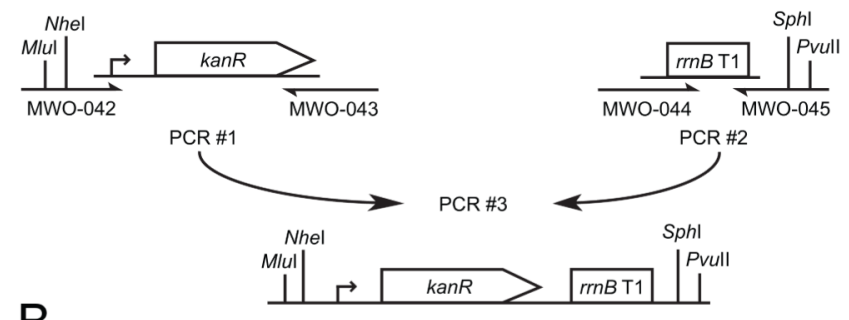

B
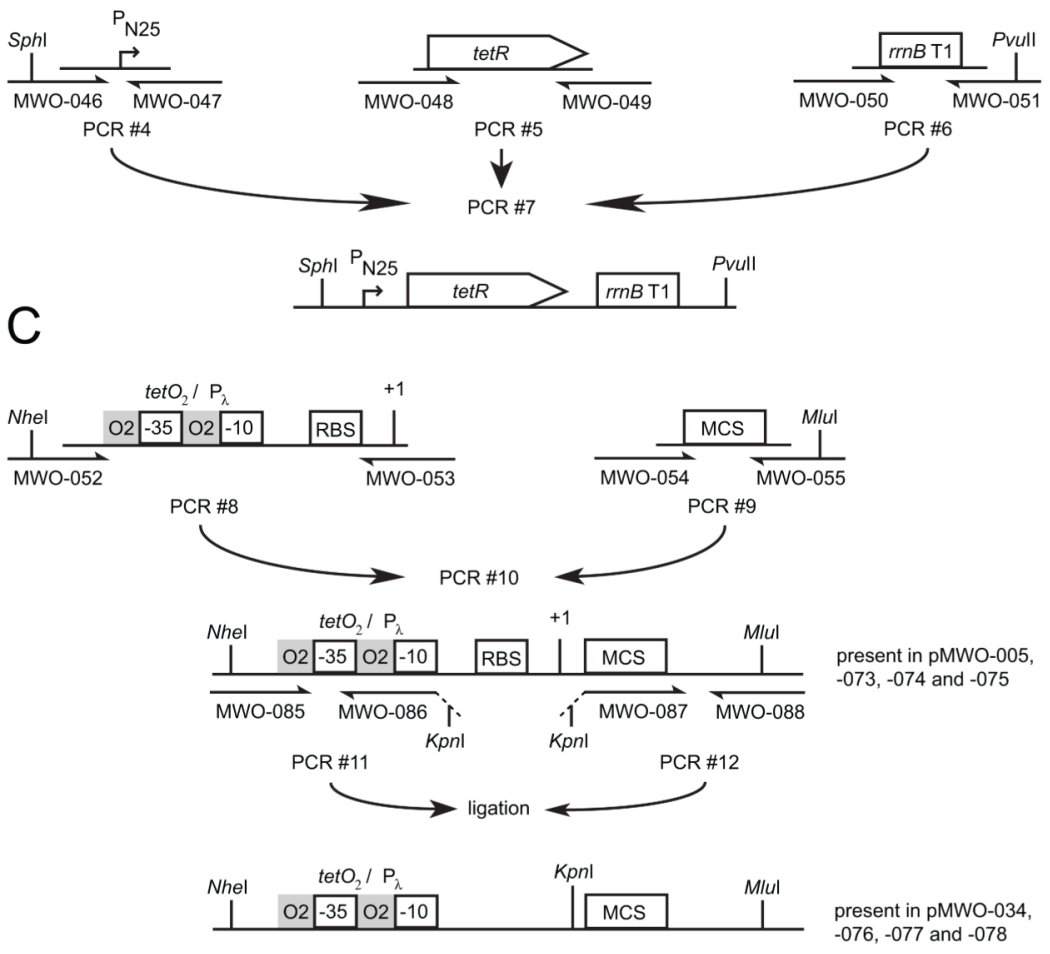

Fig. 1. PCR and cloning schemes of vector modules

(A) antibiotic resistance module. (B) tet repressor module. (C) promoter/operator and multicloning site modules. 


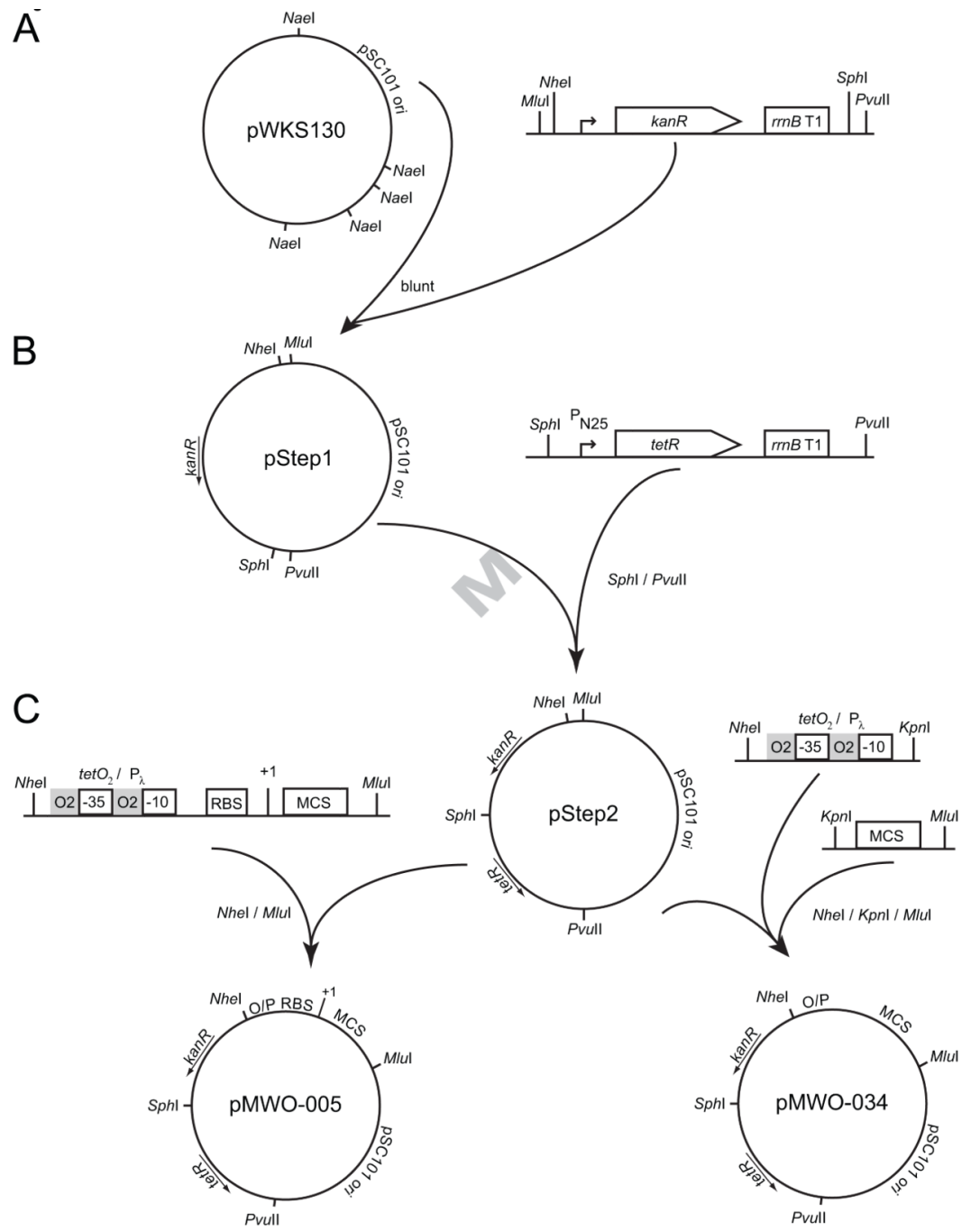

Fig. 2. Vector cloning steps

(A) Ligation of kanamycin resistance gene with pSC101 origin of replication. (B) Digestion of pStep1 and ligation of tet repressor module with pStep1. (C) Digestion of pStep2 and ligation with module containing promoter/operator sequence and multi-cloning site. 

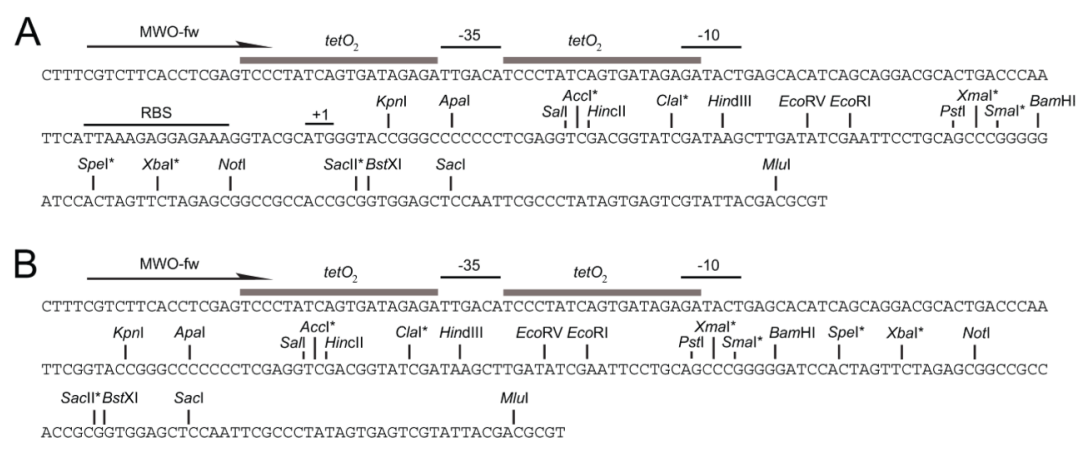

Fig. 3. Nucleotide sequence of modules containing promoter/operator and multi-cloning site (A) Module with RBS and ATG start codon. (B) Module without RBS and ATG start codon. Restriction sites marked with an asterisk are present in some vectors but not all. 

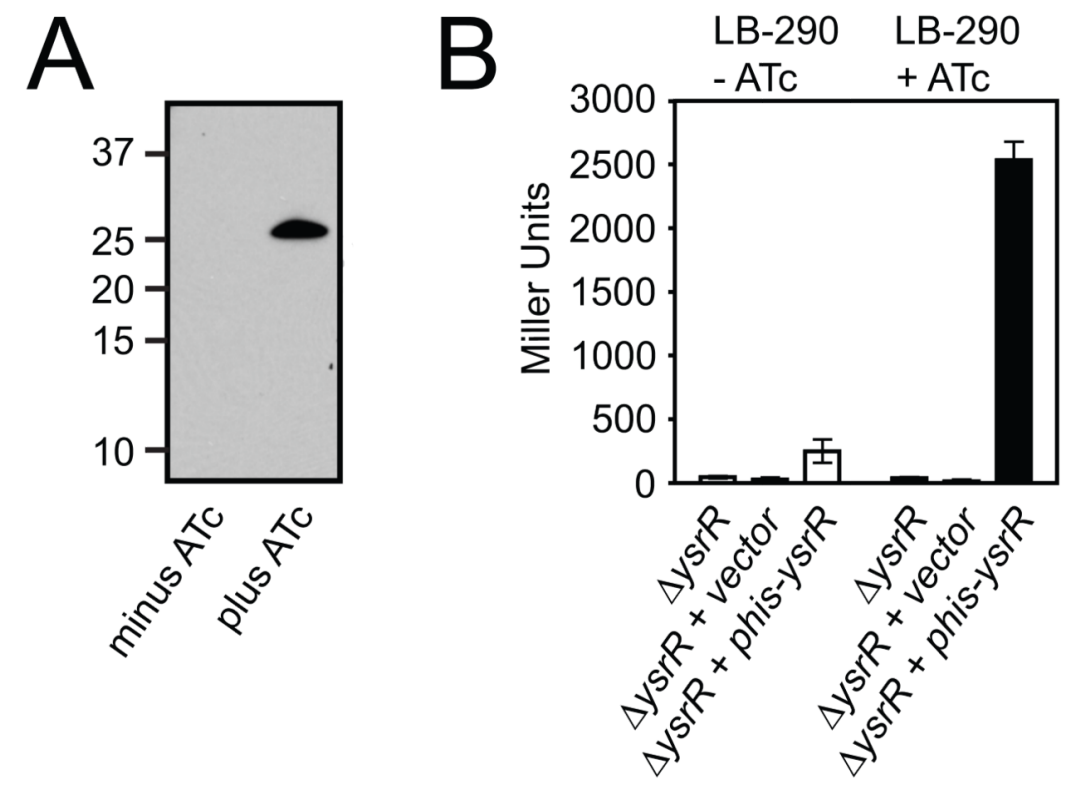

Fig. 4. Expression test of his-ysrR

(A) Immunoblot with anti-His antibodies against samples of $Y$. enterocolitica strain YVM1250 complemented with pMWO-081 encoding his-ysrR that was treated with or without the inducer ATc. Lane "minus ATc" represents a culture without induction and lane "plus ATc" shows a culture induced with $50 \mathrm{ng} / \mathrm{ml}$ ATc as described in Materials and Methods. Numbers reflect the protein standard in kDa. (B) Induction of His-YsrR expression. $\beta$-galactosidase assay with uninduced and induced $(50 \mathrm{ng} / \mathrm{ml} \mathrm{ATc})$ cultures of $Y$. enterocolitica strain YVM1250 grown in LB-290. $\Delta y s r R$ refers to YVM1250; $\Delta y s r R+$ vector refers to YVM1250 + pMWO-005; $\Delta y s r R+\mathrm{pHis}-y s r R$ refers to YVM1250 + pMWO-081. 
Table 1

Bacterial strains and plasmids

\begin{tabular}{|c|c|c|}
\hline Strain or plasmid & Relevant characteristics & Reference \\
\hline \multicolumn{3}{|l|}{ E. coli } \\
\hline DH5a & 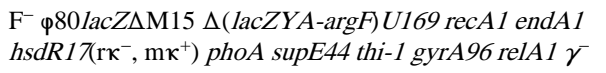 & Invitrogen \\
\hline BL21PRO & Source for $\mathrm{P}_{25 \mathrm{~N}} \&$ tet $R$ & Clontech \\
\hline \multicolumn{3}{|l|}{ Y. enterocolitica } \\
\hline JB580v & $8081 \mathrm{v}\left(\mathrm{r}^{-} \mathrm{m}^{+} \mathrm{Nal}^{\mathrm{r}}\right)$ serotype $\mathrm{O}: 8$ & (Kinder et al., 1993) \\
\hline YVM1250 & ysaE $E^{+}, y s a E: l a c Z Y A, \triangle y s r R, \mathrm{Cm}^{\mathrm{r}}$ & (Walker et al., 2010) \\
\hline \multicolumn{3}{|l|}{$Y$. pseudotuberculosis } \\
\hline IP32953 & & (Chain et al., 2004) \\
\hline \multicolumn{3}{|l|}{$Y$.pestis } \\
\hline YP6 & $\mathrm{CO} 92 \mathrm{pCD}^{-}$ & (Cathelyn et al., 2006) \\
\hline \multicolumn{3}{|l|}{ Plasmids } \\
\hline pET24b(+) & Source for $k a n R$ & Novagen \\
\hline pWKS130 & Source for pSC101 ori, multi-cloning site & (Wang and Kushner, 1991) \\
\hline pACYC184 & Source for p15A ori & (Chang and Cohen, 1978) \\
\hline p34S-Sm2 & Source for $\mathrm{Sm}^{\mathrm{r}}$ & (Dennis and Zylstra, 1998) \\
\hline pLP-PROTet-6xHN & Source for $\mathrm{P}_{\mathrm{L} \text { tetO }}$ & Clontech \\
\hline pMWO-038 & His- $y s r R$ in pMWO-005 & this study \\
\hline pMWO-081 & His- $y s r R$ in pMWO-005 & this study \\
\hline
\end{tabular}


Table 2

Oligonucleotides used in this study

\begin{tabular}{|c|c|}
\hline Name & Oligonucleotide sequence $\left(5^{\prime}-3^{\prime}\right)^{a}$ \\
\hline \multicolumn{2}{|c|}{ Sequencing primers } \\
\hline MWO-010 & GGTTTCATGCTCCGTTAAGTC \\
\hline MWO-011 & GGTTCGTTCTCATGGCTCACGC \\
\hline MWO-012 & GTTCCCTTTAGTGAGGG \\
\hline MWO-013 & GCGAAGAGGCCCGCACCG \\
\hline MWO-014 & CCCATATAAATCAGCATCC \\
\hline MWO-015 & СТССТTCATTACAGAAACGGC \\
\hline MWO-016 & GTTCCGTTGCGCTGCCCGG \\
\hline MWO-017 & GATTCTTCTCGCTTCCGGCG \\
\hline MWO-018 & GTCTCAGCCAATCCCTGGG \\
\hline MWO-025 & GGCAGTGAATGGGGGTAAATGGC \\
\hline MWO-026 & CTCAAAACCGCCCGCTTAACACC \\
\hline MWO-027 & GCGTTCCAGCTAAGGCTAAGGC \\
\hline MWO-028 & GCCTTTAGGGTTTTAAGGTCTG \\
\hline MWO-029 & GCGATTCAGGCCTGGTATGAG \\
\hline MWO-030 & CTTGCCATCCTATGGAACTGCCTCGG \\
\hline MWO-031 & GGCAATACGCACGCTTTCAGGC \\
\hline MWO-032 & GGAAAGCGGGCAGTGAGCGCAACG \\
\hline MWO-033 & GCTCTGCCAGTGTTACAACC \\
\hline MWO-034 & CAACAAAGCCACGTTGTGTCTC \\
\hline MWO-035 & CTATGCCAAGTTCTCAAGCG \\
\hline MWO-036 & CATGGTGAACAGCTTTGAATGCACC \\
\hline \multicolumn{2}{|c|}{ Primers for construction of pMWO-005 } \\
\hline MWO-042 & GGGGAAACGCGTATAGCTAGCGGAACGAAAACTCACGTTAAGGG \\
\hline MWO-043 & AAATCAAATAATGATTTTATTTTGACCATTCAAATATGTATCCGCTC \\
\hline MWO-044 & GTCAAAATAAAATCATTATTTGATTTCAATTTTGTCCCACTCCCCC \\
\hline MWO-045 & GGGGAACAGCTGATAGCATGCGGCAGCAAAACCCGTACCCTAGG \\
\hline MWO-046 & 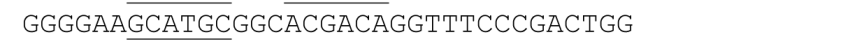 \\
\hline MWO-047 & СTTTTATCTAATCTGGACATATCAATTCGGGGCGGGATTTC \\
\hline MWO-048 & GCCCCGAATTGATATGTCCAGATTAGATAAAAG \\
\hline $\begin{array}{ll}\text { MWO-049 } & \frac{\mathrm{CT}}{\mathrm{GT}} \\
\text { MWO-050 } & \underline{\mathrm{GT}} \\
\text { MWO-051 } & \mathrm{GC} \\
\text { MWO-052 } & \mathrm{GC} \\
\text { MWO-053 } & \mathrm{CC} \\
\text { MWO-054 } & \text { GC } \\
\text { MWO-055 } & \text { GC }\end{array}$ & 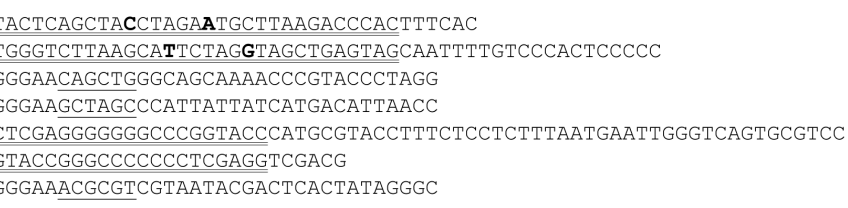 \\
\hline \multicolumn{2}{|c|}{ Primers for construction of pMWO- 034} \\
\hline MWO-085 & CGTGAGTTTTCGTTCCGCTAGCCC \\
\hline MWO-086 & CCCAAGTGGTACCGAATTGGGTCAGTGCGTCC \\
\hline MWO-087 & GGGAAGTGGTACCGGGCCCCCCCTCGAGG \\
\hline MWO-088 & GGAAACGCGTCGTAATACGACTCAC \\
\hline
\end{tabular}




\begin{tabular}{ll} 
Name & Oligonucleotide sequence $\left(\mathbf{5}^{\prime} \mathbf{-}^{\prime}\right)^{\boldsymbol{a}}$ \\
\hline MWO-137 & GGAAACGCGTGGAGTGTATACTGGCTTACTATG \\
MWO-138 & GGGGAACAGCTGACAACTTATATCGTATGG \\
MWO-139 & CTACTAGCTAGCGCGCTCGTTCGCCAGCCAGG \\
MWO-140 & GGGGAAGCATGCTTATTTGCCGACTACCTTGG \\
MWO-142 & CTATTATACAGAAAAATTTTCC \\
MWO-143 & GGCAAAGCCGTTTTTCCATAGG \\
MWO-144 & GCTGAGTAGCAATTTTGTCC \\
Primers for construction of pMWO-038 and pMWO-081 \\
MWO-072 & GGCAAGTTCTAGATTATAGAGAAATTTCATGAGC \\
MWO-147 & CCGAAGTGGTACCAAATGCATCACCATCACCATCACACACAAACGAAAACGCTC \\
MWO-150 & GGGAAGTGGTACCATCACCATCACCATCAC \\
General sequencing primers for vector series \\
MWO-fw & CGTCTTCACCTCGAGTCC \\
MWO-rv-1 & CAGCCTGAAACAGGCGATGCTGC \\
MWO-rv-2 & GTATCACATATTCTGCTGACGC \\
\hline
\end{tabular}

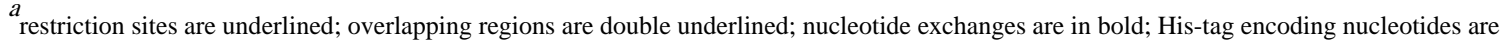
underlined with dashes 
